\title{
On magnetospheric transmissivity of cosmic rays
}

\author{
K. KudelA \\ Institute of Experimental Physics, Slovak Acad. Sci., \\ Watsonova 47, 04353 Košice, Slovakia \\ I.G. Usoskin \\ Sodankylä Geophysical Observatory, University of Oulu, \\ PO-3000, FIN-90014 Oulu, Finland
}

Received 30 June 2003; final version 12 September 2003

\begin{abstract}
Results of computations of cosmic-ray trajectories in the model magnetic field obtained for a high-latitude neutron-monitor station (Oulu) and a middle-latitude one (Lomnický Štít) are summarized. The method is slightly different from previous computations by controlling the smoothness of the trajectory. At the lowest rigidity edge of allowed trajectories for Oulu (above the atmospheric cut-off), the external field and the addition of Dst shows variations of the asymptotic directions with the level of geomagnetic activity and with local time of observations. The transmissivity function introduced here, accounting for variability of the magnetic field with changing $K p$ and with local time, may be used as a reference for description of the average magnetospheric transparency at middle latitudes and it is probably better approximation than IGRF only. There are indications of appearance of windows of allowed trajectories at very low rigidities in the middle latitudes at $500 \mathrm{~km}$ (altitude of, e.g., CORONAS satellites or ISS) during the geomagnetic disturbance which is not the case for trajectories computed from the ground.
\end{abstract}

PACS: 96.40.Kk, 95.85.Ry

Key words: cosmic rays, magnetosphere

\section{Introduction}

Details of cosmic-rays transport in the Earth's magnetosphere, to access ground cosmic-ray stations or low-altitude satellites, have been studied since long (see, e.g., $[1,2])$. The main tool for this study is a numerical back-tracing of cosmic-ray particle's trajectory in a geomagnetic field model. The trajectory starts from the observational site and is traced back by reversing the particle's velocity vector and the sign of charge. The computed trajectory is regarded as allowed (A), if it crosses the magnetospheric boundary, and as forbidden $(\mathrm{F})$, if the trajectory rests on the Earth's surface, or trapped, if it remains within the magnetosphere for a long time. Progress of cosmic-ray trajectory computations in the geomagnetic fields has been summarized recently in [3]. Between the allowed and forbidden ranges of particle's rigidity, there is an area of complicated intermittent structure, the penumbra. From the penumbra structure, the following concepts of cut-off rigidity can be identified [4]: lower cut-off, $R_{\mathrm{L}}$, is the rigidity, below which all trajectories are forbidden, upper cut-off, $R_{\mathrm{U}}$, is the rigidity above which all trajectories are allowed, and 
the effective rigidity cut-off, $R_{\mathrm{C}}$. It is usual to characterize a given site by its vertical effective cut-off. The vertical cut-off rigidities computed for various epochs in the internal geomagnetic field are summarized in [5]. In addition to the internal geomagnetic field, external field models were used for the trajectory computations of cosmic rays in several papers [6-8]. Introducing an external magnetic field model, which assumes the varying current systems within the magnetosphere and near its boundary regions, provides a better approach to the description of the cut-offs, the structure of penumbra and of the asymptotic directions.

Sometimes the classical approach fails to explain observational facts. For example, it has been found recently that there is a significant difference in count rates of NMs with close asymptotic directions (e.g. Oulu and Apatity) during an anisotropic phase of GLE that occurred during 14-16 hours of the local magnetic time [9]. Including the external field for low-altitude satellites improves the agreement between computed and measured positions of cut-off latitudes, although a difference of $\approx 1^{\circ}$ still remains [10]. Therefore, computations of trajectories, using different approaches, are still important for description and understanding the magnetospheric transmissivity of cosmic rays.

In this paper, we introduce a method of trajectory computations and compare it with earlier results. We describe effects of the external-field model [11] and its extension with including Dst, according to [12], which is found to be relevant for neutron monitor measurements at high (Oulu) and a middle (Lomnický Štít) latitude stations as well as for low-altitude satellites. We also test the stability of the trajectory and consequent cut-off computations vs. uncertainties of the magnetic-field models. The transmissivity function introduced here can be used as an additional reference to the concepts of lower, effective and upper cut-offs at middle latitudes. For example, such an approach has been recently applied for determining cut-off rigidities for the low-orbiting International Space Station [13].

\section{The calculation method}

The model magnetic field used here is a superposition of the DGRF model including all harmonics up to the order of 10 and of the Tsyganenko'89 externalfield model [11] (Ts89 in the following) according to the numerical scheme [14]. The magnetospheric boundary is taken as follows: the shape of the magnetopause is approximated by the form of [15] for the day side $\left(X_{\mathrm{GSM}}>0\right)$, while the border is fixed at the distance of 25 Earth radii for the night side $\left(X_{\mathrm{GSM}}<0\right)$. The calculated asymptotic directions are given in GEO coordinate system. Using the magnetopause model on the day side gives the information whether the traced CR particle accesses the magnetosphere from the day side or not. The night side boundary is taken as a sphere of $25 R_{\mathrm{E}}$ radius similarly to earlier calculations by other authors (e.g., $[2,16]$ used $25 R_{\mathrm{E}}$ boundaries for all sectors of local time). In such an approach there is, however, a discontinuity at $X=0$. For each crossing of $X=0$ between two subsequent points of the trajectory, we distinguish the sense of the direction: if $X>0$ at the latter point then the distance is compared to 
$25 R_{\mathrm{E}}$, and if $X<0$, the position is checked with respect to the magnetopause day side model. The IGRF field model used here is adopted from [17] and from http://nssdc.gsfc.nasa.gov/space/models/igrf.html.

The transport equation of a charged particle in the geomagnetic field is solved numerically by Runge-Kutta method of the sixth order. The elementary computational step along the trajectory is taken as $2 \pi r / n$, where $r$ is the particle's gyroradius (in the local magnetic field) and $n$ is initially taken as 100 , similarly to the procedure in [18]. During computations, we control both the conservation of the particle's velocity modulus and smoothness of the trajectory. If the deviation of $\boldsymbol{v}$ (velocity vector) in two subsequent points of the trajectory exceeds a preselected value $(T)$ at some elementary computational step, then this step is recalculated with the halved length. If the trajectory is not resolved as F or A after the preselected number $N$ of elementary computational steps, then it is regarded as forbidden one (F). Then the value of $R_{\mathrm{C}}$ is computed from the system of $\mathrm{F}$ and $\mathrm{A}$ trajectories. Backward computations start from the point with the altitude of $20 \mathrm{~km}$ above the observational site in vertical (radial to the Earth's center) direction assuming the spherical shape of the Earth with the average radius $6371.2 \mathrm{~km}$. As an example, Table 1 shows the effective vertical cut-off rigidities computed using IGRF only, for Oulu and Lomnický Štít for different values of $N$ when $T$ is fixed in comparison to the results given in [5] for the epoch 1985.

Table 1. The effective vertical cut-off rigidities (in GV) for two stations (Oulu, $65.05^{\circ}$ $\mathrm{N}, 25.47^{\circ} \mathrm{E}$; Lomnický Štít $49.20^{\circ} \mathrm{N}, 20.22^{\circ} \mathrm{E}$ ), epoch 1985 , computed using IGRF model only, with different maximum number of computational steps $N$ for $T=10^{-3}$ rad. The last column is from Table 1 of [5].

\begin{tabular}{|l|c|c|c|c|c|c|c|}
\hline \multirow{2}{*}{ Station } & \multicolumn{5}{|c|}{$N$} & \multirow{2}{*}[5]{} \\
\cline { 2 - 7 } & $2.5 \times 10^{4}$ & $5 \times 10^{4}$ & $10^{5}$ & $2 \times 10^{5}$ & $5 \times 10^{5}$ & $10^{6}$ & \\
\hline Oulu & 0.85 & 0.83 & 0.79 & 0.77 & 0.76 & 0.76 & 0.76 \\
\hline Lomnický Štít & 4.25 & 4.16 & 4.10 & 3.96 & 3.94 & 3.94 & 3.95 \\
\hline
\end{tabular}

Although these methods are based on different numerical schemes of trajectory tracing, there is a good agreement in the effective vertical cut-offs. One can see that increasing $N$ above $2 \times 10^{5}$ results in only a small additional decrease of the calculated cut-offs, due to resolving a few more low-rigidity quasi-trapped trajectories as allowed ones. Moreover, the upper cut-off value $R_{\mathrm{U}}$ is stable for both stations for $N>10^{5}$. Therefore, we use $N=2 \times 10^{5}$ in the following.

\section{Transmissivity function}

Details of the magnetospheric transparency for cosmic rays can be described by a transmissivity function $T F(R, L T, K p, D)$, where $R, L T, K p$, and $D$ are the particle's rigidity, local time, $K p$ index, and the day of the year, respectively. In paper 
[8] the authors examined the seasonal variation of the cut-offs for Lomnický Štít and found that it is much less than the diurnal variability of the cut-offs (Fig. 2 of the cited paper illustrates the cut-offs for March, June, September, and December, 1990). Accordingly we neglect the seasonal variation. The transmissivity function $(T F)$ is defined as the probability of a primary particle with rigidity in the interval $[R, R+\mathrm{d} R]$ to access vertically the position above the measurement site at a given local time $L T$ during the time, when geomagnetic activity is characterized by $K p$ index. $T F$ is calculated as the ratio of the number of $\mathrm{A}$ to $(\mathrm{A}+\mathrm{F})$ trajectories within the rigidity interval $[R, R+\mathrm{d} R]$. We note that, although calculation of each trajectory is unambiguous in the frames of the deterministic computational model, some uncertainties and random fluctuations are always present in the real magnetosphere. The transmissivity function is defined in a probability sense which is more natural taking into account the real uncertainties. Usually, the count rate of a given neutron monitor (NM) is given as follows:

$$
N=\int_{R_{\mathrm{C}}}^{\infty} G(R) \cdot Y(R) \cdot \mathrm{d} R,
$$

where $G(R)$ and $Y(R)$ are the differential spectrum of $C R$ at the Earth's orbit and the specific yield function of NM, $R$ is the particle's rigidity, and $R_{\mathrm{C}}$ is the effective rigidity cut-off. Using the transmissivity function, the count rate is now given as

$$
N=\int_{0}^{\infty} T F \cdot G(R) \cdot Y(R) \cdot \mathrm{d} R
$$

Hofer and Flückiger [19] introduced in similar manner the geomagnetic filter function in analysing a Forbush decrease. While Eqs. (1) and (2) yield very similar results on average, it appears important to use the transmissivity function approach in some particular cases as will be discussed later. We also note that the difference between Eqs. (1) and (2) may be significant for solar cosmic rays with a soft energy spectrum.

Using different elementary steps in rigidity for the trajectory computations $(\Delta R$ ranging from $10^{-3}$ to $\left.10^{-5} \mathrm{GV}\right)$ in the interval $(0.4-0.8) \mathrm{GV}$, we found that refining the rigidity resolution below $10^{-4} \mathrm{GV}$ does not significantly change the shape of $\mathrm{TF}$ and for practical purposes the value of $\Delta R=10^{-3} \mathrm{GV}$ is sufficient [20].

\section{Asymptotic directions for a high-latitude station}

The transmissivity of cosmic rays through magnetosphere to the Oulu neutron monitor $\left(65.05^{\circ} \mathrm{N}, 25.47^{\circ} \mathrm{E}\right)$ was calculated. We note that the vertical effective cutoff rigidity of Oulu NM is close to the atmospheric cut-off assuming the response function of a neutron monitor near the sea level (e.g. [21,22]). Figure 1 depicts the fine structure of asymptotic directions within the penumbra at the position of Oulu. The fine structure of penumbra and its implications for high-latitude NM count rate was studied also in [23]. Although the structure of penumbra at high latitudes is not expected to affect significantly the count rate of a ground neutron 

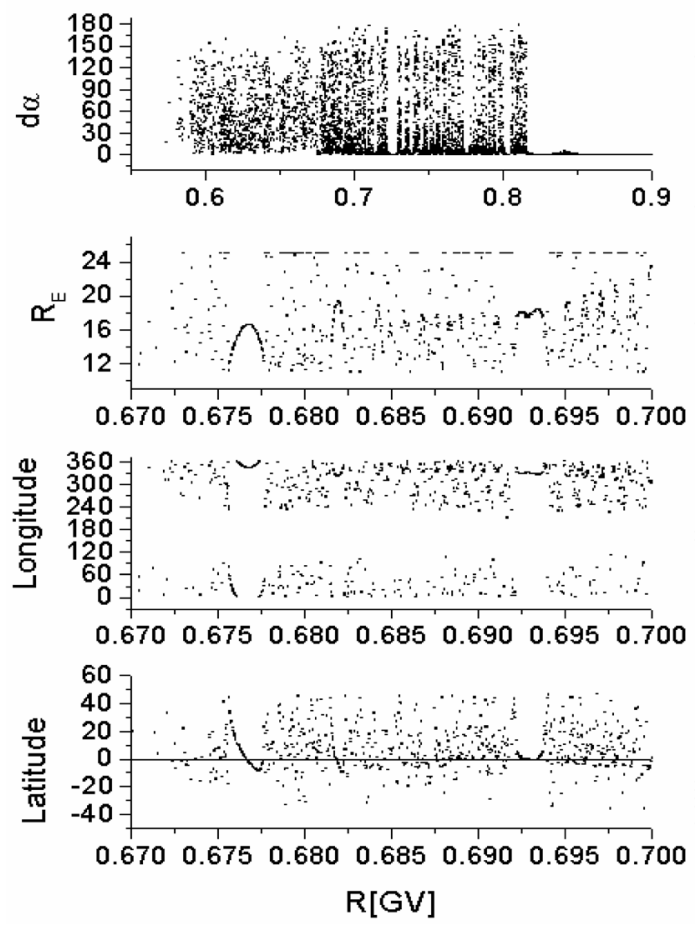

Fig. 1. Asymptotic directions computed for Oulu position with the rigidity step $\Delta R=$ $10^{-5} \mathrm{GV}$, as for January 21, 1986, $12 \mathrm{UT}$, low $\mathrm{Kp}$ (IOPT $=1$ in Ts89 model). The upper panel shows the angular difference between the asymptotic directions of the two consecutive allowed trajectories. The lower three panels display the distance to the entry point in $R_{\mathrm{E}}$, and asymptotic longitude and latitude defined by Shea and Smart, 1975.

monitor, its implications for energetic particle measurements onboard low-altitude polar orbiting satellites may be important.

One can see from the top panel of Fig. 1 that the neighbouring allowed trajectories are spread widely and randomly in their asymptotic directions as $\mathrm{d} \alpha$ is large and has no regular pattern vs. $R$ at $R<0.675$. The divergence of the neighbouring trajectories, $\mathrm{d} \alpha$, is not reduced with increasing $\Delta R$, which implies that the model gets unstable. For rigidities in the range from $0.75 \mathrm{GV}$ to $0.81 \mathrm{GV}$, many trajectories are close to each other (small $\mathrm{d} \alpha$ values), but these regular structures are intermitted by areas with large divergence of the neighbouring trajectories. We have studied this transition (from random to regular patterns of trajectories) range in more details. Lower panels of Fig. 1 show the asymptotic directions for the rigidity range (0.67-0.7) GV. One can see that among random large fluctuations there are intervals of rigidity with regular smooth changes of the asymptotic direction (e.g. $(0.676-0.677)$ or $(0.692-0.694) \mathrm{GV})$. These intervals correspond to the entry in the day side sector, while intervals of chaotic behaviour of the asymptotics are mostly 
related to the night side entries. Similar intervals of regular changes were found also in the penumbra for the mid-latitude station (Lomnický Štít). This fact is likely related to a more complicated character of low-rigidity particles' trajectories in the geomagnetic tail and to a more complicated geometry of the night side magnetosphere [24] than analytical models predict. Limitations of empirical magnetic-field models have been mentioned earlier (see, e.g. [25,26] and references therein). In particular, large fluctuations of the observed values of $B_{z}$ near the neutral sheet of magnetosphere with respect to the model value have been reported even during times of low geomagnetic activity $(K p<2)$.

We have estimated the effect of uncertainties in the magnetic-field modelling on the cosmic ray trajectories computation for the Oulu position for two cases: one with $\left(B_{z}+10\right) \mathrm{nT}$ and the other one with $\left(B_{z}-2\right) \mathrm{nT}$ in the tail central region for $X<-10 R_{\mathrm{E}}$, which corresponds to the spread of experimental points in Fig. 4 of [26]. The geomagnetic conditions were fixed to quiet ones $(I O P T=1)$. The values IOPT used here are taken according to the model (Tsyganenko, 1989): IOPT $=1$ for $K p=0,0+, I O P T=2$ for $K p=1-, 1,1+, I O P T=3$ for $K p=2-, 2,2+$, $I O P T=4$ for $K p=3-, 3,3+, I O P T=5$ for $K p=4-, 4,4+$, and $I O P T=6$ for $K p \geq 5-$. Calculations were done for two fixed times (12 and $00 \mathrm{UT}$ ), and no notable changes in the values of $R_{\mathrm{L}}, R_{\mathrm{C}}$, nor $R_{\mathrm{U}}$, related to the spread of $B_{z}$, were found. However, the employed variations of $B_{z}$ resulted in significant changes of the asymptotic directions for many trajectories. For the midnight, this affects only particles with the rigidity below $0.734 \mathrm{GV}$, and changes of asymptotic directions range from $30^{\circ}$ to $80^{\circ}$. The effect of changing $B_{z}$ is much more significant during the noontime as it affects rigidities of up to $1.2 \mathrm{GV}$. This may even be significant for the ground-based observations of an anisotropic flux of cosmic rays (see e.g. [9]). For better understanding of the night side particle entry the geomagnetic tail model should be extended to larger distances than the border adjusted here (and in earlier computations by other authors).

We have studied also variations of the effective vertical cut-off rigidity vs. the time of observations and vs. the level of geomagnetic activity. We performed a set of calculations for different times with 2-hour step for low $(I O P T=1)$ and high $(I O P T=6)$ level of geomagnetic activity as well as for the two levels of Dst. The

Table 2. Effective vertical cut-offs (GV) for Oulu calculated using the Ts89 field model and with Dst extension by method [11] added to IOPT $=6$. Date is January 21, 1986.

\begin{tabular}{|c|c|c|c|c|}
\hline \multirow{2}{*}{$\mathrm{UT}$} & \multicolumn{4}{|c|}{ Magnetic activity } \\
\cline { 2 - 5 } & $I O P T=1$ & $I O P T=6$ & $D s t=-100 \mathrm{nT}$ & $D s t=-300 \mathrm{nT}$ \\
\hline 00 & 0.619 & 0.252 & 0.129 & 0.051 \\
06 & 0.672 & 0.487 & 0.407 & 0.197 \\
12 & 0.647 & 0.413 & 0.346 & 0.169 \\
18 & 0.610 & 0.253 & 0.159 & 0.051 \\
\hline
\end{tabular}


rigidity step was fixed to $\Delta R=10^{-3} \mathrm{GV}$. The results are shown in Table 2 . In all computations, where the epoch is not explicitly mentioned, the internal field is taken for January 21, 1986.

An increase of the geomagnetic activity causes a depression of the calculated cut-off. The smallest depression was found around $06 \mathrm{UT}$ in all cases, while the deepest one is expected in the afternoon and night sectors. The computations indicate that most of the trajectories, which change their status from forbidden (or quasi-trapped) at low geomagnetic activity to allowed at high activity, enter the magnetosphere from the night side. The asymptotics are concentrated eastwards of
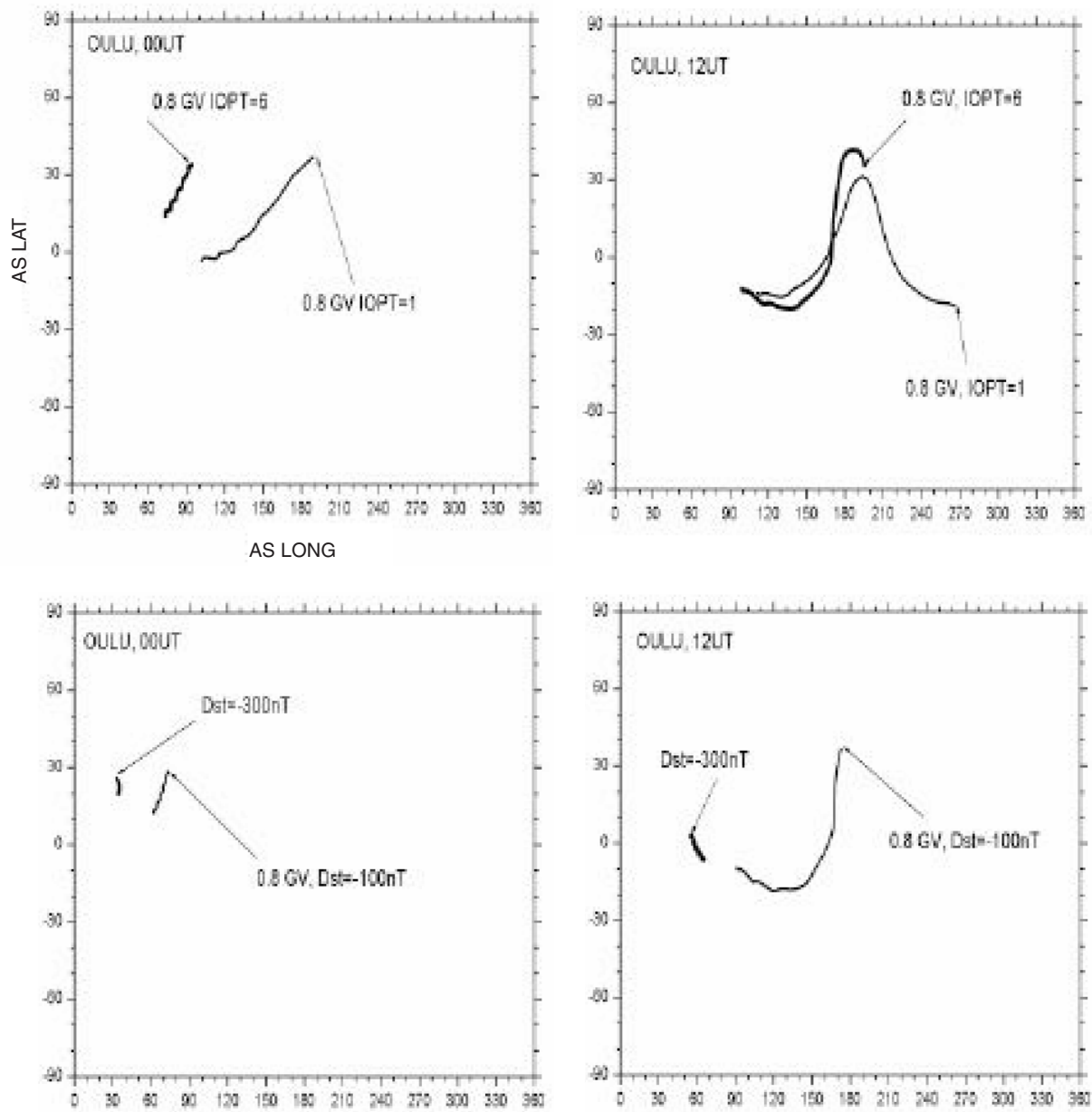

Fig. 2. Asymptotic directions of (0.8-1.5) GV primary cosmic rays accessing vertically the position of Oulu NM at midnight and noon for different geomagnetic conditions. The date is January 21,1986 . The arrows depict the asymptotic directions corresponding to

$0.8 \mathrm{GV}$ in all cases. Rigidity $1.5 \mathrm{GV}$ corresponds to the other end of the line. 
the station's site, making access more difficult during the local morning. However, since the depressed rigidity cut-off is well below the atmospheric cut-off (about $0.8 \mathrm{GV}$ ), an increase of the magnetospheric transparency during geomagnetic disturbances should not affect the count rate of a high-latitude neutron monitor, contrary to low- and middle-latitude stations. A clear illustration of this effect is the geomagnetic storm on March 30-31, 2001, when a large increase of count rate associated with the decrease of their cut-offs was found at middle- and low-latitude stations, while the count rate of high-latitude stations in both hemispheres (Oulu and Sanae) was continuously decreasing, corresponding to the time profile of the intensity of primaries outside the magnetosphere [27].

Even though cut-off depression during geomagnetic storms is insignificant for high-latitude stations, geomagnetic activity variations influence the asymptotic directions at the low-rigidity range above the atmospheric cut-off (Fig. 2).

For low geomagnetic activity $(I O P T=1)$, the asymptotic directions are only slightly changed with the local time except for a north-south asymmetry seen at the lowest $R$ in noon-midnight comparison. However, the asymptotic directions shift significantly westwards and their range is narrowed with increasing geomagnetic activity, especially in the midnight sector. The shift in longitude is more than $90^{\circ}$ for $I O P T=6$ and exceeds $150^{\circ}$ for $D s t=-300 \mathrm{nT}$. These effects, especially the narrowing of the acceptance direction interval during strong geomagnetic disturbances, should be taken into account when analysing anisotropic fluxes of low-energy cosmic rays, as, e.g., for GLE on May 24-25, 1990 [28]. The effect of the slight variations of model $\boldsymbol{B}$, of both additive and multiplicative character, was examined in [20].

\section{The effect of external field at middle latitudes}

At middle latitudes, where the atmospheric cut-off for quiet geomagnetic conditions is negligible compared to the geomagnetic one, the magnetospheric transparency for cosmic rays changes with the geomagnetic activity and with local time of observations. We have analysed a structure of TF for Lomnický Štít in great details for a wide range of parameters: calculations were performed for different local times with the step of 1 hour as well as for 6 different levels of the geomagnetic activity parameter (IOPT) in Ts89 model. The variation of $R_{\mathrm{C}}$ and $R_{\mathrm{U}}$ depicts a wavy structure for each IOPT over a day with the maximum at (7-8) UT and minimum at (20-21) UT. The value of the cut-off rigidity decreases with increasing $I O P T$ at all local times [8]. The transmissivity functions computed for different IOPT averaged over 24 hours are displayed in Fig. 3. The seasonal variability of cut-offs for Lomnický Štít was found to be lower than the diurnal one [8] and thus it is not studied here.

One can see that TF changes significantly with varying geomagnetic activity. The weighted transmissivity function which accounts for the frequency of different level disturbances occurrence can be introduced as a reference for the long-term average geomagnetic activity. The probability of occurrence of events with various IOPT is shown in Fig. 4 for 1980-1990. The transmissivity function weighted 

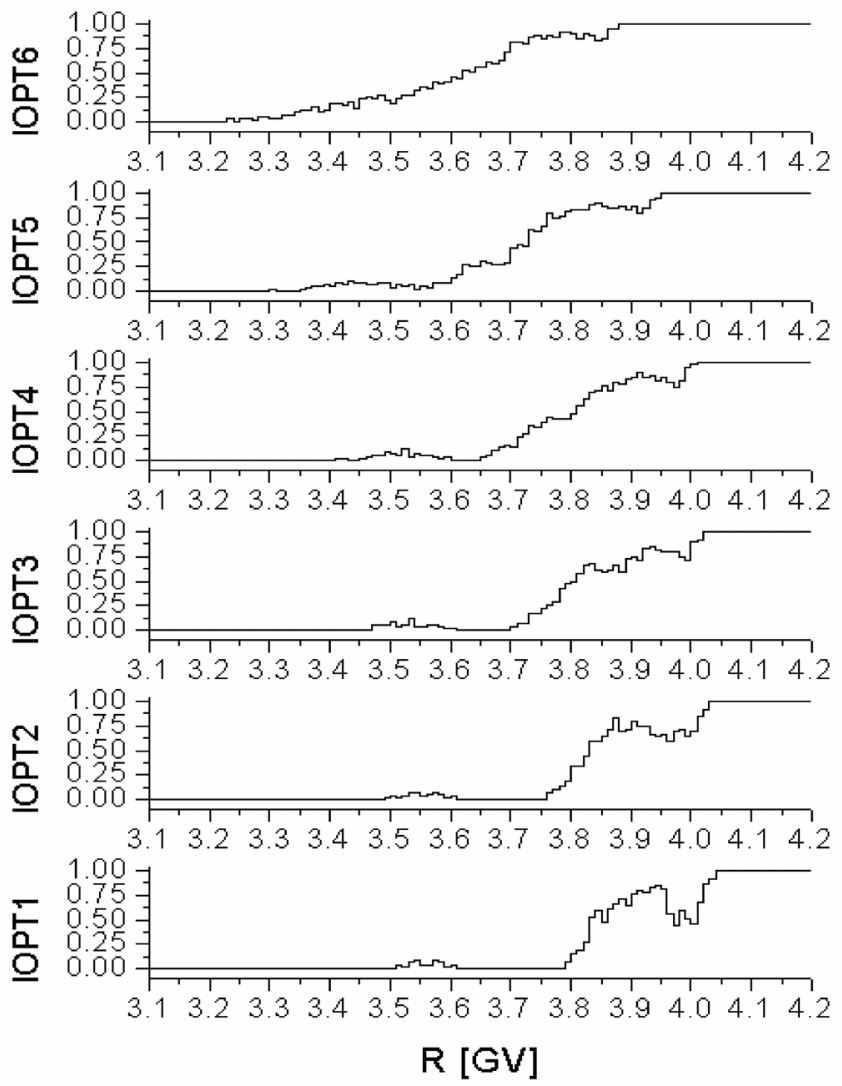

Fig. 3. The transmissivity functions for Lomnický Štít NM, 24-hour averaged, calculated using the Ts89 model for different levels of geomagnetic activity.

over the probability of geomagnetic disturbances occurrence is shown in Fig. 4, too. Long-term averaged values of the vertical cut-offs for Lomnický Stít, obtained through such weighted averaging, are $R_{\mathrm{L}}=3.466 \mathrm{GV}, R_{\mathrm{U}}=3.926 \mathrm{GV}$, and $R_{\mathrm{C}}=3.802 \mathrm{GV}$. Although secular change of the geomagnetic field is small for Lomnický Śtít location, the approach of the weighted transmissivity function should be considered with some caution if used for the long term studies of cosmic rays, since the geomagnetic activity is changing with the solar cycle.

In some cases the time of observations should be taken into account. For instance, an increase in count rates of a number of middle- and low-latitude stations was observed during morning hours associated with the very strong geomagnetic disturbance ( $K p$ up to $9-$ ) on March 31, 2001. However, high-latitude stations (Oulu and Sanae) did not observe this increase. In order to understand this situation, we have estimated the expected NM response using Eq. (2) and TF calculated for this particular time. The calculated change of $T F$ is so large (see Fig. 5) that 

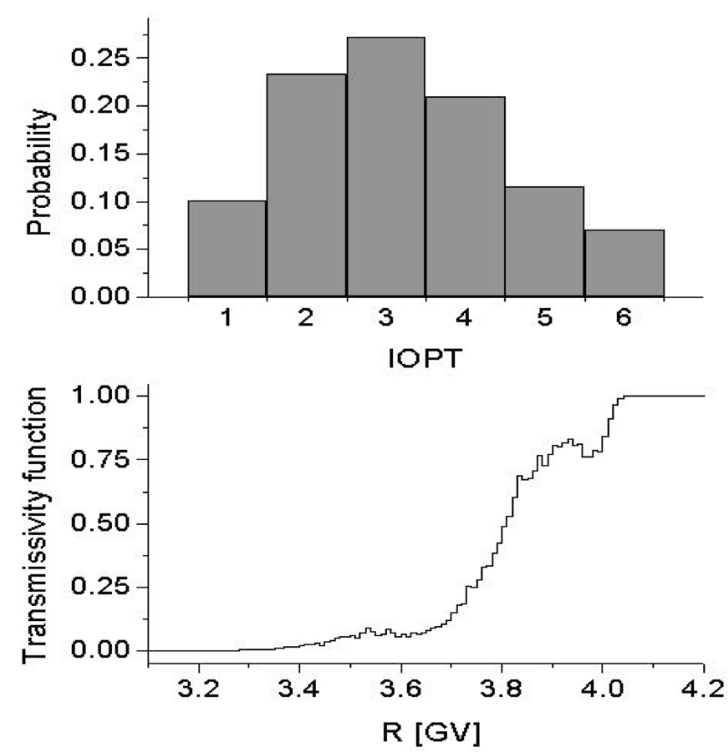

Fig. 4. Upper panel: probability of occurring of various IOPT during the period 1980-1990 (histogram from data available at http://nssdc.gsfc.nasa.gov/omniweb). Lower panel: The transmissivity function for Lomnický Štít weighted by the probabilities displayed in the upper panel.

an increase of the count rate expected at middle- and low-latitude stations is in accord with observations. We note that the Ts89 model employs only $K p$ index with 3 -hour resolution with the upper bound of $I O P T=6$. However, the profile of middle-latitude NM count rates corresponds to the measured Dst profile, especially for those stations having asymptotics in the night sector [27]. The increasing Dst decreases significantly the rigidity cut-off and modifies $T F$ shifting it towards lower rigidities. The results of computations for two levels of $D$ st are shown in Table 3 and the transmissivity function affected by Dst is shown in Fig. 5 .

Table 3. Effective vertical cut-offs (in GV) for Lomnický Štít in Ts89 field model and with $D s t$ extension by method [11] added to $I O P T=6$.

\begin{tabular}{|c|c|c|c|c|}
\hline \multirow{2}{*}{$\mathrm{UT}$} & \multicolumn{4}{|c|}{ Magnetic activity } \\
\cline { 2 - 5 } & $I O P T=1$ & $I O P T=6$ & Dst $=-100 \mathrm{nT}$ & Dst $=-300 \mathrm{nT}$ \\
\hline 00 & 3.871 & 3.491 & 3.353 & 2.626 \\
06 & 3.892 & 3.691 & 3.564 & 2.981 \\
12 & 3.877 & 3.663 & 3.573 & 2.851 \\
18 & 3.860 & 3.532 & 3.329 & 2.563 \\
\hline
\end{tabular}




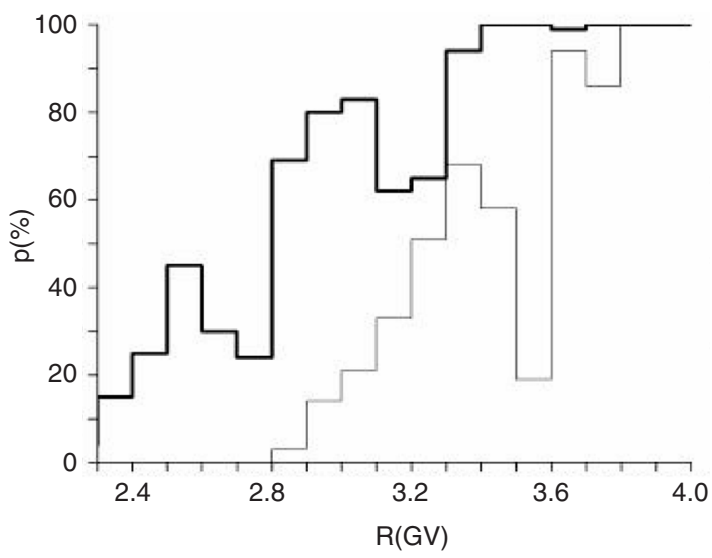

Fig. 5. Transmissivity function for Lomnický Štít 06UT with $D s t=-100 \mathrm{nT}$ (thin line) and $D s t=-358 \mathrm{nT}$ (corresponding to minimum Dst on March 31, 2001). IOPT $=6$.

The azimuthal and local time dependence of cosmic ray intensity variations due to geomagnetic cut-off changes, especially at middle latitudes, were investigated in [29-31]. In the former of cited papers the vertical cut-off rigidities and their changes were determined by utilizing trajectory-tracing technique in the magnetic field modelled as a simple dipole field to which the disturbance is superposed. Here we have computed expected changes of asymptotic directions for various local times and geomagnetic activity levels using the Ts89 field model. Figure 6 depicts the results for the low-rigidity part of cosmic ray primaries contributing to the count rate of Lomnický Štít NM. For the selected interval of rigidities, the computations show a rather stable structure where the neighbouring asymptotic directions are smoothly organized.

When the geomagnetic activity is low, only slight variations of the asymptotic longitude with the local time are expected, and the studied rigidity interval of 0.5 GV width corresponds to a wide longitudinal interval of asymptotics. An increase of the activity level leads, similarly to higher latitudes, to a westward shift and to shrinking of the asymptotics longitudinal extent. The asymptotics are shifted by more than $210^{\circ}$ for $4 \mathrm{GV}$ particles at midnight, when the activity is changed from $I O P T=1$ to $I O P T=6$ with $D s t=-300 \mathrm{nT}$. The shifts are slightly smaller for the daytime observations.

An experimental test of TF changes can be done either by a neutron-monitor latitude surveys or by measurements onboard low-altitude polar orbiting satellites. A real survey requires long time during which geomagnetic field can be significantly changed. However, a correlative analysis of data from neutron monitors located nearly at the same meridian over the entire range of latitudes and thus forming a meridianal chain (e.g., Oulu - Kiel - Lomnický Štít - Jungfraujoch - Rome Israel - South Africa - Antarctica) would be also regarded as a latitude survey. The latter approach has been recently performed in [10] using measurements of energetic 

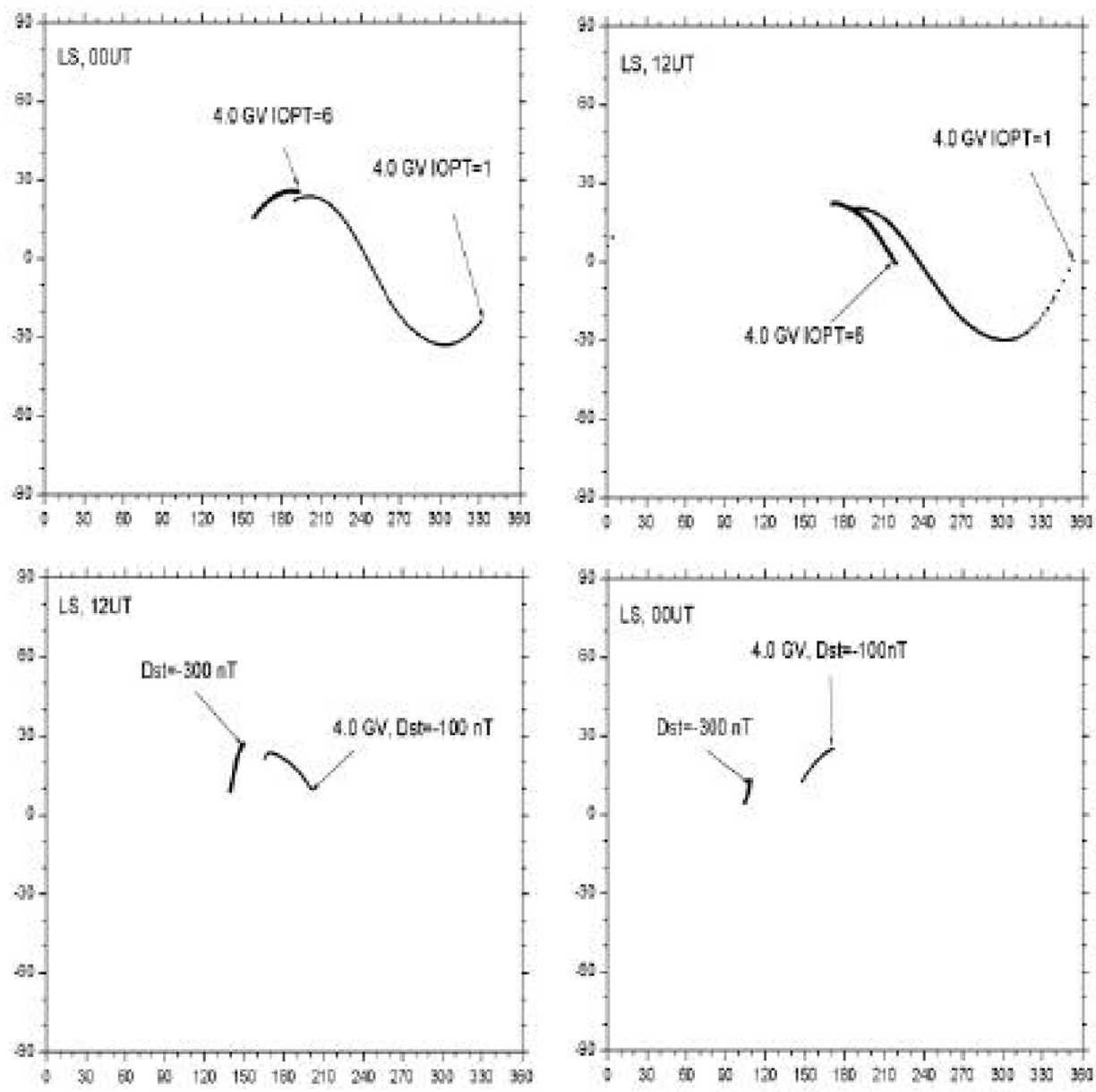

Fig. 6. Asymptotic directions of (4.0-4.5) GV primary cosmic rays accessing vertically Lomnický Śtít position at midnight and noon for different geomagnetic conditions. The epoch for internal field is January 21, 1986. The arrows correspond to asymptotic directions at $4.0 \mathrm{GV}$ at each line.

solar protons on SAMPEX [32]. It was concluded that more detailed dynamical models of the geomagnetic field are needed to obtain a better correspondence to inspace particle measurements. Recently [33] illustrated that measurements of solar particles onboard a low-altitude polar orbiting satellite, which provide an indication of the geomagnetic cut-off location four times per orbital period, are useful for possible warning of significant cut-off suppression. Most probably a single global parameter of magnetic activity adopted by models (like Dst) is not sufficient to explain the full details of observations. A good possibility to experimentally study changes of $T F$ is provided by the SONG instruments onboard the low-altitude 
polar orbiting satellite CORONAS I. SONG measures, along with neutrons and gamma rays, protons with the energy above $50 \mathrm{MeV}$ [34]. Its high count rate allows to study variations of low-energy cosmic rays at different latitudes separately for each orbit, providing thus nearly momentary latitudinal survey. Kuznetsov et al. [35] reported that, while being in agreement at high latitudes, SONG data did not correspond to the data of middle-latitude neutron monitors (Climax and LARC, both have $P_{\mathrm{C}} \approx 3 \mathrm{GV}$ ) during a strong geomagnetic disturbance and the related
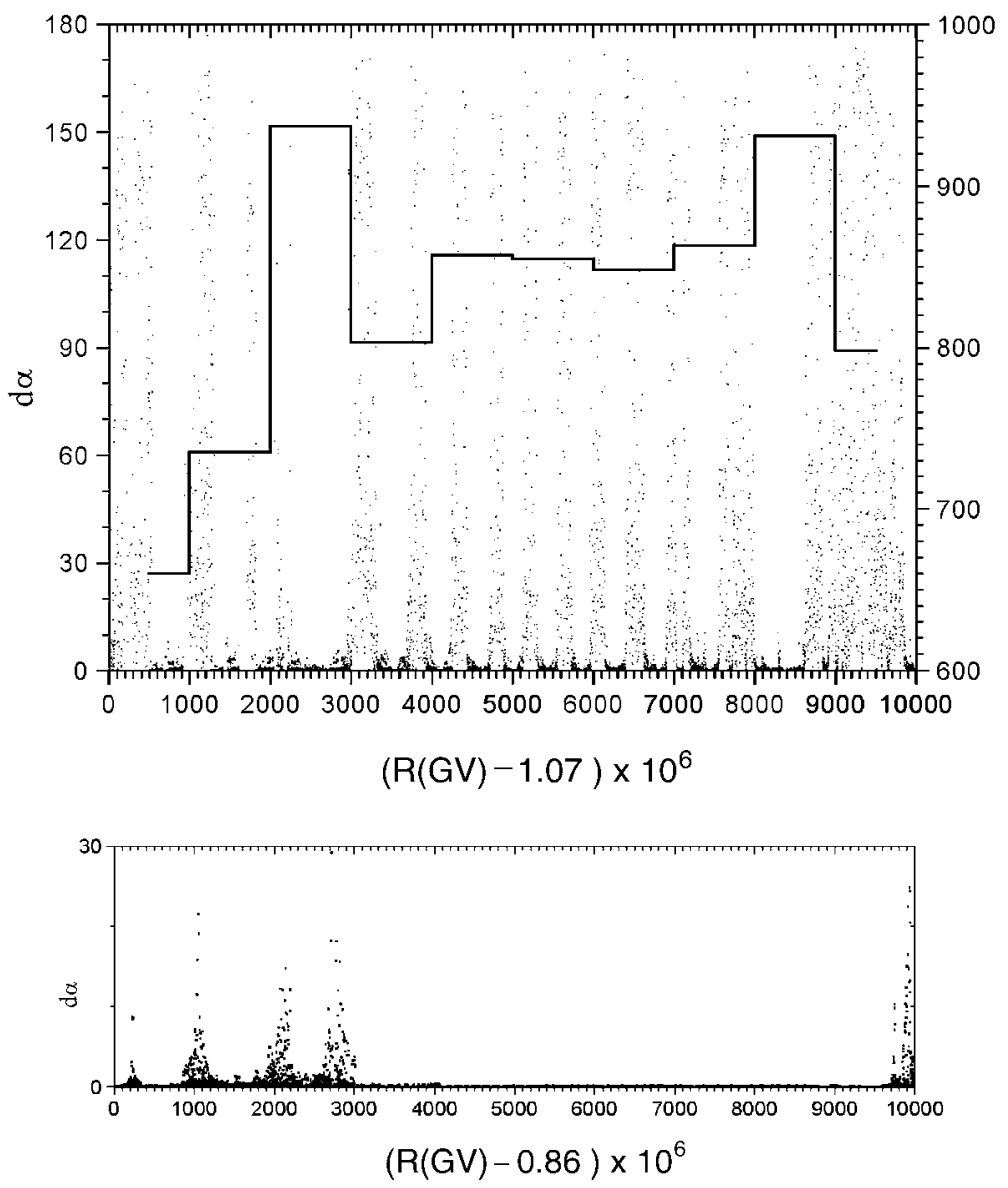

Fig. 7. Fine structure of the penumbra at the altitude of $500 \mathrm{~km}$ above the position of LARC neutron monitor calculated using the Ts89 model with Dst extension $-300 \mathrm{nT}$. Rigidity intervals of (1.07-1.08) GV (upper panel) and (0.86-0.87) GV (lower panel) are depicted. The angular difference $\mathrm{d} \alpha$ of two subsequent asymptotic directions is displayed by dots (labeled left axis, in degrees, similar value as in Fig. 1). Solid line and the right axis in the upper panel correspond to the computed transmissivity function. $T F=1$ for the lower panel. 
Forbush decrease on April 17, 1994. While neutron monitors at middle latitudes did not record notable variations, SONG observed a significant increase of CR at the same $L$ values as Climax and LARC during the maximum $D$ st depression. We have computed trajectories of cosmic rays for the location of the LARC station $\left(-62.20^{\circ} \mathrm{S}, 301.04^{\circ} \mathrm{E}\right)$ both for the ground (altitude $20 \mathrm{~km}$ ) and for a low orbit (500 km, corresponding to CORONAS I). We found that a large Dst depression $(-300 \mathrm{nT})$ may cause a very strong depression of the effective vertical cut-off rigidity from $3 \mathrm{GV}$ down to $1.71 \mathrm{GV}$ and to $0.92 \mathrm{GV}$ for the altitudes of $20 \mathrm{~km}$ and $500 \mathrm{~km}$, respectively. The computations were done with the rigidity step of $10^{-5} \mathrm{GV}$. The fine structure of the both penumbras is rather complicated. Narrow windows of allowed trajectories with stable asymptotic directions were found for the $500 \mathrm{~km}$ altitude. These windows correspond to particles entering the magnetosphere from the night side. Two samples of the penumbra are shown in Fig. 7. Interestingly, the interval of lower rigidities (0.86-0.87) GV (shown in the bottom panel of Fig. 7) has a very regular structure and the transmissivity function (equal to 1) in contrast to the other, more energetic, interval of (1.07-1.17) GV (upper panel). No such transparency windows were found for the altitude of $20 \mathrm{~km}$.

Such windows of transparency may result in the count rate increase observed at the satellite altitude, but missed by the ground neutron monitor. The transparency window shown in Fig. 7 is narrow (0.01 GV in rigidity) and the effect of this window alone is rather small. However, this example stresses that the transmissivity of magnetosphere for low-energy particles may be very different at different altitudes, even at the same geographical position, during periods of high geomagnetic activity. This indicates that a broader case study of the inconsistency between the "orbit by orbit profile" of a low-altitude satellite crossing the fixed mid-latitude $L$-shell and the ground-based NM profile at the same $L$ (as e.g. Fig. 3 of [35]) during geomagnetic disturbance and Forbush decrease) would be of interest from the point of view of penumbra expectations and approaches of the trajectory calculations.

\section{Summary}

Using the method of trajectory computations of cosmic rays in the model geomagnetic field, with the self-adjusted control of the elementary computational step by keeping the "smoothness" of the trajectory, we study details of the geomagnetic cut-off penumbra for two cosmic ray stations: middle-latitude Lomnický Štít and high-latitude Oulu. The complicated structure of the penumbra, computed with high rigidity resolution, implies that intervals of smooth change of asymptotic directions with rigidity correspond mainly to trajectories entering the day-side magnetopause, while night entry is sometimes seen as chaotic changes of the asymptotic directions. The asymptotic directions on the night side are influenced by variations of $B_{z}$ in the neutral sheet of the geomagnetic tail. The changes of the cut-off and narrowing of the asymptotic range with the magnetic activity and local time should be taken into account for the study of low-energy cosmic-ray anisotropy in interplanetary space or of the anisotropy of solar energetic particles during GLEs for 
high-latitude stations.

The transmissivity function introduced here accounting for variability of the magnetic field with changing $K p$ and with local time may be used as an additional reference (to the $R_{\mathrm{L}}, R_{\mathrm{C}}, R_{\mathrm{U}}$ concept) for description of the average magnetospheric transparency at middle latitudes.

It was found that the structure of penumbra and the corresponding transmissivity function can be significantly different for the ground-based observations and for low-altitude polar orbiting satellites during strong geomagnetic disturbances. As we have shown qualitatively, this effect can be responsible for the observed difference between cosmic-ray intensity as measured by ground-based stations and by low-altitude polar orbiting satellites. A correlative study of data from neutron monitors (meridional chain) and low-altitude polar satellites with large geometrical factors for high-energy particles would provide a tool for testing the transparency expectations based on computations of trajectories.

KK wishes to thank the LAPBIAT program and the Sodankylä Geophysical Observatory/Oulu University for support as well as VEGA grant No. 1147.

\section{References}

[1] K.G. McCracken, U.R. Rao, and M.A. Shea: Technical Report 77 (NYO-2670), MIT, Laboratory for Nuclear Science, Cambridge (MA), USA, 1962.

[2] M.A. Shea and D.F. Smart: Report AFCRL-TR-75-0177, Air Force Cambridge Res. Lab., Hanscom (MA), USA, 1975, p. 100.

[3] D.F. Smart, M.A. Shea, and E.O. Flückiger: Space Sci. Rev. 93 (2000) 305.

[4] D.J. Cooke, J.E. Humble, M.A. Shea, D.F. Smart, N. Lund, I.L. Rasmussen, B. Byrnak, P. Goret, and N. Petrou: Nuovo Cimento C 14 (1991) 213.

[5] M.A. Shea and D.F. Smart: in Proc. 27th ICRC, Hamburg, 2001, Vol. 10, p. 4036.

[6] E.O. Flückiger, and E. Kobel: J. Geomag. Geoelectr. 42 (1990) 1123.

[7] O.A. Danilova and M. Tyasto: in Proc. 24th ICRC, Rome, 1995, Vol. 4, p. 1066.

[8] K. Kudela, M. Storini, P. Bobik, and J. Kassovicova: in Rayos Cosmicos 98 (Ed. J. Medina), Universidad de Alcala, Alcala de Henares, 1998, p. 71.

[9] E.V. Vashenyuk et al.: in Proc. 27th ICRC, Hamburg, 2001, Vol. 8, p. 3383.

[10] D.F. Smart and M.A. Shea: Adv. Space Res. 28 (2001) 1733.

[11] N.A. Tsyganenko: Planet. Space Sci. 37 (1989) 5.

[12] P.R. Boberg, A.J. Tylka, J.H. Adams, E.O. Flückiger, and E. Kobel: Geophys. Res. Lett. 22 (1995) 1133.

[13] P. Bobik, M. Boschini, M. Gervasi, K. Kudela, and P.G. Rancoita: ESA publication series, ESA (2003 - in press).

[14] M. Peredo and N.A. Tsyganenko: GEOPACK93, package of FORTRAN programs, 1993.

[15] D.G. Sibeck, R.W. McEntire, A.T.Y. Lui, R.E. Lopez, S.M. Krimigis, R.B. Decker, L.J. Zanetti, and T.A. Potemra: Geophys. Res. Lett. 14 (1987) 1011. 
[16] M.A. Shea, D.F. Smart, and K.G. McCracken: J. Geophys. Res. 70 (1965) 4117.

[17] R.A. Langel: Eos Trans. AGU 73 (1992) 182.

[18] J. Kassovicova and K. Kudela: Preprint of IEP SAS, 1998-01, Kosice, 1998.

[19] M. Hofer and E.O. Flückiger: J. Geophys. Res. 105 (2000) 23.

[20] P. Bobik, K. Kudela, and I. Usoskin: in Proc. 27th ICRC, Hamburg, 2001, Vol. 10, p. 4056.

[21] K. Nagashima, S. Sakakibara, K. Murakami, I. Morishita: Nuovo Cimento 12 (1989) 173.

[22] L.I. Dorman, G. Villoresi, N. Iucci, M. Parisi, M.I. Tyasto, O.A. Danilova, and N.G. Ptitsyna: J. Geophys. Res. 105 (2000) 21,047.

[23] V.V. Pchelkin and E.V. Vashenyuk: Izv. AN, ser. Fiz. 65 (2001) 416.

[24] T.I. Pulkkinen and N.A. Tsyganenko: J. Geophys. Res. 101 (1996) 27,431.

[25] D.P. Stern and N.A. Tsyganenko: Eos Trans. AGU 73 (1992) 489; 493.

[26] M. Peredo, D.P. Stern, and N.A. Tsyganenko: J. Geophys. Res. 98, No. A9 (1993) 15,343 .

[27] K. Kudela and M. Storini: in European Space Agency Proc. SOLSPA 2001, SP-477, 2002, p. 289.

[28] Y. Fedorov, M. Stehlik, K. Kudela, and J. Kassovicova: Sol. Phys. 208 (2002) 325.

[29] E.O. Flückiger, D.F. Smart, and M.A. Shea: J. Geophys. Res. 88 (1983) 6961.

[30] S. Kudo, M. Wada, P. Tanskanen, and M. Kodama: J. Geophys. Res. 92, No. A5, 4719 .

[31] E.O. Flückiger, D.F. Smart, and M.A. Shea: J. Geophys. Res. 91 (1986) 7925.

[32] R.A. Leske, R.A. Mewaldt, E.C. Stone, and T.T. von Rosenvinge: in Proc. 25th ICRC, Durban, 1997, Vol. 2, p. 381.

[33] R.A. Leske, R.A. Mewaldt, E.C. Stone, and T.T. von Rosenvinge: J. Geophys. Res. 106, No A12 (2001) 30,011.

[34] J. Balaz, A.V. Dmitriev, M.A. Kovalevskaya, K. Kudela, S.N. Kuznetsov, I.N. Myagkova, J.I. Nagornych, J. Rojko, and S.P. Ryumin: in Proc. Int. Astron. Union Colloquium (Coronal structures), Tatranská Lomnica, Slovakia, Sept. 20-24, 1993 (Eds. V. Rušín, P. Heinzel, and J.-C. Vial), Veda, Bratislava, 1994, p. 635.

[35] S.N. Kuznetsov, I.N. Myagkova, S.P. Ryumin, K.Kudela, R. Bucik, and H. Mavromichalaki: J. Atmos. Solar-Terrestrial Phys. 64 (2002) 535. 